CORRECTIONS

\title{
John Clement Charlton
}

In the process of shortening the length of this Obituary ( $B M J$ 2013;347:f6250, doi:10.1136/bmj.f6250) for the print issue, we miscounted the number of surviving relatives. The last sentence of the print version, which appears in the "Section PDF" online, should have read: "He leaves his sister, three sons, eight grandchildren, and one great grandchild."

Cite this as: BMJ 2014;348:971

(๑) BMJ Publishing Group Ltd 2014 